\title{
Reduced Risk of Cancer in Patients with Bipolar Affective Disorder
}

\author{
Eyal Rosenshfir ${ }^{1}$, Pnina Shemesh ${ }^{1}$, Dov Aizenberg ${ }^{2}$, Yoram Barak ${ }^{3}$ \\ ${ }^{1}$ Hadassa Ein-Karem, Jerusalem, Israel; ${ }^{2}$ Geha Mental Health Center, Petah-Tikva, Israel; ${ }^{3}$ Abarbanel Mental Health Center, Bat-Yam, \\ Israel. \\ E-mail: mdybarak@Gmail.com
}

Received January $12^{\text {th }}, 2011$; revised February 25 ${ }^{\text {th }}, 2011$; accepted March $9^{\text {th }}, 2011$.

\begin{abstract}
Background: research focusing on incidence of cancer amongst bipolar affective disorder (BAD) is sparse. Aim: to examine the incidence of cancer in a cohort of BAD inpatients. Methods: the study sample was comprised of BAD patients who had been admitted to a tertiary care mental health center during the period: 1990 to 2006. The data for the sample was cross-referenced with the National Cancer Registry. Analyses of Standardized Incidence Rates (SIR) for all organ systems malignancies were performed. Results: of 1,638 BAD patients included in the present study, 72 (0.04\%) were diagnosed as suffering from co-morbid malignancy. This reflects a significantly reduced risk; SIR of 0.27 (95\% CI 0.21-0.34). Reduced risk of cancer held true for both males and females ( 0.20 and 0.34 , respectively). Nineteen women developed breast cancer; SIR $=0.42$ (95\% CI 0.25-0.66). Conclusions: the present study demonstrates reduced rates of cancer amongst patients suffering from BAD—with special reduction in breast cancer.
\end{abstract}

Keywords: Bipolar Affective Disorder, Cancer, Tertiary Care.

\section{Introduction}

The unique epidemiological characteristics of mentally ill patients suffering from comorbid malignancies might shed light on the explicit etiology of cancer and contribute to prevention. The interest in cancer amongst the mentally ill is further due to the need to provide better services to this underprivileged population. Rates and incidence of malignancy are different amongst patients suffering from severe mental disorders compared to general population rates [1]. These observations have mostly been ascribed to patients suffering from serious mental illness - notably schizophrenia and their first degree relatives $[2,3]$.

Recently it has been reported that bipolar affective disorder (BAD) patients have increased incidence of several physical diseases such as: Cardiovascular, diabetes, hepatitis and alcohol dependence. On the other hand lower incidence of hyperlipidemia, lymphomas and metastatic cancer was reported by the same group [4]. A large population based study of BAD patients compared cancer risks of 6 common cancers (breast, colon, rectal, gastroesophageal, prostate, and respiratory) to patients with schizophrenia who have similar lifestyle characteristics but who are likely to differ with respect to use of medication and any inherent physical correlates of the disease [5]. Patients with BAD in said study had similar cancer risks as people without mental disorders. Despite several shared factors-biological, medication [6], episodes of hospitalizations and unhealthy behavior-there is limited recent research on the association for bipolar disorder $[7,8]$.

The aim of the present study was to examine the incidence of cancer in a regional cohort of BAD inpatients in Israel.

\section{Methods}

All adult inpatients who were admitted to the Abarbanel Mental health Center during the period 1990 to 2006 were screened for possible inclusion in the present analysis. In line with the Israeli Ministry of Health's regulations, all HMO's in the center's catchment area are obliged to refer patients to this center. There is no charge for psychiatric inpatient treatment emphasizing that there is little referral bias in the present sample. The Abarbanel Mental Health Center is a university affiliated tertiary care institute serving a dense urban catchment area with a population of 870,000 subjects.

Computerized medical records at our center were used 
to categorize all adults who had been inpatients at any time during the study period. Inclusion criteria were: age (older than 18 years) and a diagnosis of bipolar affective disorder (DSM criteria). Thus, we included every patient with an episode of mania (present or lifetime). All subjects records in the sample were linked to the National Cancer Registry of Israel in order to calculate and compare cancer rates. Cancer incidence rates in this cohort of bipolar patients were compared for the same time interval with cancer rates recorded for age matched Jewish population of Israel.

National recording and reporting of cancer cases to the Israeli Center for Disease Control (ICDC) is obligatory since 1960 [9]. All included patients' computerized data was meshed with records of the Israeli National Cancer Registry; part of the ICDC to identify pathologically validated co-morbid malignancies.

We thus identified adult bipolar patients with pathologically confirmed cancers (International Classification of Diseases, 9th Revision, CM), diagnosed from Jan., 1990 and until Dec., 2006. Additional information was gathered for patients age, gender, age at cancer onset and type of cancer, if any.

The Abarbanel Mental Health Center IRB approved the study.

\subsection{Statistical Analysis}

To compare standardized cancer incidence ratios (SIR) in patients suffering from bipolar affective disorder with the general population we based our calculations of expected ratios on the general adult Jewish population of Israel. For purposes of the present analysis, patients were considered to be at risk until one of the following events: cancer onset, death or end of follow-up on Dec., 2006.

\section{Results}

Of the 1,638 BAD patients' records analyzed, 655 (40\%) were female and $983(60 \%)$ male. Mean age for the group was $47.2+22.6$; range $18-91$ years. It is of interest to note that the majority of patients were free of physical co-morbidity, 1,045 (64\%). The three most common physical disorders in this sample were: cardiovascular 233 (14\%), metabolic/diabetes 93 (6\%) and endocrine 41 (3\%).

Seventy-two BAD patients were diagnosed as suffering from cancer as reflected by the National Cancer Registry. The overall rate of malignancy in this sample is low compared to the general population; computed SIR 0.27 (95\% CI, 0.21-0.34). The reduced risk of cancer held true for both male patients (SIR 0.20; 95\% CI, 0.13-0.29) and female patients (SIR 0.34; 95\% CI, 0.25-0.45). See Table 1 for details.

Amongst 983 male BAD patients, 25 (2.5\%) had de- veloped cancer. Among 655 female BAD patients, 19 (2.9\%) developed breast cancer which was the most prevalent of cancer types in females, and 28 (4.3\%) developed cancers of other type. The SIR for breast cancer amongst female patients was significantly reduced compared to the general female population, 0.42 (95\% CI, 0.25-0.66).

Following correction for multiple testing controlling for the False Discovery Rate (FDR) colon, breast and overall (all sites) cancer rates were significantly reduced, see Table 1 [11].

\section{Discussion}

The present study suggests that BAD patients may have a reduced rate of cancer. As schizophrenia and bipolar disorder may be the outcomes of shared causative processes with the co-morbidity between disorders mainly due to additive genetic effects [10] the effects on cancer risk are expected to be shared in part. Thus, it is not surprising that BAD patients in the relatively homogenous population of Israel have reduced risk of cancer similar to that reported for schizophrenia [12].

A recent Israeli publication [13] also focusing on the risk of cancer among patients with BAD reported an overall enhanced cancer risk in both genders. Breast cancer risk in their report was not significantly different than that in the general female population. Our findings differ from the BarChana group suggesting a significant reduction in cancer rate in $\mathrm{BAD}$ patients. As both studies are based on an Israeli sample it is interesting to note differences in methodology. First, the BarChana group included only Israeli-born subjects while we did not use place of birth as a criteria. A national Israeli mental health survey found that the largest group of immigrants, those from the former Soviet Union, have higher rates of mood disorders [14]. In addition, the health of immigrants is often better than the general population given the "healthy immigrant effect". Thus, the inclusion of immigrants in the present study could have resulted in a sample with higher rates of bipolar disorder and lower rates of cancer. Second, we included only patients diagnosed with BAD while manic (non-organic) state comprised more than $10 \%$ of their sample. Third, we included patients hospitalized only after 1990 thus may be reflecting a healthier cohort and finally, we included on patients admitted to psychiatric tertiary care center.

It is possible that some cancers were undiagnosed in people BAD perhaps owing to underreporting of physical problems, lack of participation in screening programs or denial of physical morbidity due to manic and hypomanic coping. Such factors would tend to bias the risk of cancer downward. Furthermore, the present study focused on patients presenting with a very severe course of 
Table 1. SIR (95\% CI) by cancer site-Cancer morbidity amongst BAD inpatients $\mathrm{N}=1,638$.

\begin{tabular}{|c|c|c|c|c|c|}
\hline Cancer site & Observed & Expected & SIR & Lower & Upper \\
\hline Mouth (C04-C06.9) & 1 & 0.63 & 1.60 & 0.02 & 8.89 \\
\hline Esophagus (C15-C15.9) & 1 & 1.52 & 0.66 & 0.01 & 3.65 \\
\hline Stomach (C16-C16.9) & 2 & 6.40 & 0.31 & 0.04 & 1.13 \\
\hline Small intestine (C17-C17.9) & 1 & 0.79 & 1.26 & 0.02 & 7.03 \\
\hline Colon (C18-C18.9) & 4 & 21.27 & 0.19 & 0.05 & 0.48 \\
\hline Rectum (C19-C20.9) & 3 & 9.91 & 0.30 & 0.06 & 0.88 \\
\hline Larynx (C32-C32.9) & 1 & 2.38 & 0.42 & 0.01 & 2.34 \\
\hline Lung (C34-C34.9) & 7 & 16.55 & 0.42 & 0.17 & 0.87 \\
\hline Melanoma (C44, morphology 872..., 874...) & 1 & 17.15 & 0.06 & 0.00 & 0.32 \\
\hline Skin other (C44, other morphology) & 2 & 4.73 & 0.42 & 0.05 & 1.53 \\
\hline Breast (C50-C50.9) & 19 & 45.86 & 0.41 & 0.25 & 0.65 \\
\hline Cervix uteri (C53-C53.9) & 2 & 5.15 & 0.39 & 0.04 & 1.40 \\
\hline Corpus uteri (C54-C54.9) & 2 & 6.28 & 0.32 & 0.04 & 1.15 \\
\hline Ovary (C56-C56.9) & 3 & 5.96 & 0.50 & 0.10 & 1.47 \\
\hline Prostate (C61.9) & 2 & 21.59 & 0.09 & 0.01 & 0.33 \\
\hline Testis (C62-C62.9) & 2 & 1.20 & 1.67 & 0.19 & 6.02 \\
\hline Kidney (C64.9-C68.9) & 4 & 6.33 & 0.63 & 0.17 & 1.62 \\
\hline Bladder (C67-C67.9) & 3 & 16.05 & 0.19 & 0.04 & 0.55 \\
\hline Brain (C72-C71.9) & 3 & 10.65 & 0.28 & 0.06 & 0.82 \\
\hline Thyroid (C73-C73.9) & 1 & 5.68 & 0.18 & 0.00 & 0.98 \\
\hline Leukemia (morphology 97423,99413-98003) & 1 & 4.97 & 0.20 & 0.00 & 1.12 \\
\hline Multiple myeloma (morphology 97323,97313) & 1 & 2.91 & 0.34 & 0.00 & 1.91 \\
\hline Non Hodgkins (95903-95953,96703-97293,97403,97413) & 2 & 10.99 & 0.18 & 0.02 & 0.66 \\
\hline Other lymph node (C77-C77.9,C42-C42.9) & 1 & 2.27 & 0.44 & 0.01 & 2.45 \\
\hline Unknown (C80-C80.9) & 3 & 7.66 & 0.39 & 0.08 & 1.14 \\
\hline All & 72 & 265.30 & 0.27 & 0.21 & 0.34 \\
\hline
\end{tabular}

Legend: Abbreviations: $\mathrm{BAD}$ = bipolar affective disorder; SIR = standared incidence rate; $\mathrm{CI}$ = confidence interval. In bold are statistically significant reductions in SIR $(\mathrm{p}<0.05)$.

BAD and may not be representative of all BAD patients.

In conclusion, the present study highlights the complexity of studying cancer co-morbidity in psychiatric inpatients while suggesting that the major psychosesschizophrenia and bipolar disorder-confer a reduced cancer risk due to yet unknown mechanisms.

\section{REFERENCES}

[1] J. A. Pandiani, M. M. Boyd, S. M. Banks and A. T. Johnson, "Elevated Cancer Incidence among Adults with Serious Mental Illness,” Psychiatric Services, Vol. 57, No. 7, 2006, pp. 1032-1034. doi:10.1176/appi.ps.57.7.1032

[2] Y. Barak, T. Levy, A. Achiron and D. Aizenberg, "Breast
Cancer in Women Suffering from Serious Mental Illness,” Schizophrenia Research, Vol. 102, No. 1-3, 2008, pp. 249-253. doi:10.1016/j.schres.2008.03.017

[3] V. S. Catts, S. V. Catts, B. I. O’Toole and A. D, "Frost. Cancer Incidence in Patients with Schizophrenia and Their First-Degree Relatives-A Meta-Analysis,” Acta Psychiatrica Scandinavica, Vol. 117, 2008, pp. 323-336. doi:10.1111/j.1600-0447.2008.01163.x

[4] C. P. Carney and L. E. Jones, "Medical Co-Morbidity in Women and Men with Bipolar Disorders: A Population-Based Controlled Study," Psychosomatic Medicine, Vol. 68, No. 5, 2006, pp. 684-691. doi:10.1097/01.psy.0000237316.09601.88

[5] J. Hippisley-Cox, Y. Vinogradova, C. Coupland and C. Parker, "Risk of Malignancy in Patients with Schizophre- 
nia or Bipolar Disorder: Nested Case-Control Study,” Archives of General Psychiatry, Vol. 64, No. 12, 2007, pp. 1368-1376. doi:10.1001/archpsyc.64.12.1368

[6] Y. Cohen, A. Chetrit, Y. Cohen, P. Sirota and B. Modan, "Cancer Morbidity in Psychiatric Patients: Influence of Lithium Carbonate Treatment,” Medical Oncology, Vol. 15, No. 1, 1998, pp. 32-36. doi:10.1007/BF02787342

[7] K. R. Krishnan, "Psychiatric and Medical Comorbidities of Bipolar Disorder,” Psychosomatic Medicine, Vol. 67, 2005, pp. 1-8. doi:10.1097/01.psy.0000151489.36347.18

[8] J. S. Wang, C. L. Wang, J. F. Wen, Y. J. Wang, Y. B. Hu and H. Z. Ren, "Lithium Inhibits Proliferation of Human Esophageal Cancer Cell Line Eca-109 by Inducing a G(2)/M Cell Cycle Arrest," World Journal of Gastroenterology, Vol. 14, 2008, pp. 3982-3989. doi:10.3748/wjg.14.3982

[9] A. B. Sabin, "Proceedings: Cancer Research in Israel: Work of Academic Institutions and National Cancer Registry," National Cancer Institute Monograph, Vol. 40, 1974, pp. 57-62.

[10] P. Lichtenstein, B. H. Yip, C. Björk, Y. Pawitan, T. D. Cannon, P. F. Sullivan, et al., "Common Genetic Determinants of Schizophrenia and Bipolar Disorder in Swed- ish Families: A Population-Based Study,” Lancet, Vol. 373, No. 9659, 2009, pp. 234-139. doi:10.1016/S0140-6736(09)60072-6

[11] Y. Benjamini and Y. Hochberg, "Controlling the False Discovery Rate (FDR): A Practical and Powerful Approach to Multiple Testing," Journal of the Royal Statistical Society, Vol. 57, 1995, pp. 289-300.

[12] Y. Barak, A. Achiron, M. Mandel, I. Mirecki and D. Aizenberg, "Reduced Cancer Incidence among Patients with Schizophrenia,” Cancer, Vol. 104, No. 12, 2005, pp. 2817-2821. doi:10.1002/cncr.21574

[13] M. BarChana, I. Levav, I. Lipshitz, I. Pugachova, R. Kohn, A. Weizman, et al., "Enhanced Cancer Risk among Patients with Bipolar Disorder,” Journal of Affective Disorders, Vol. 108, No. 1-2, 2008, pp. 43-48. doi:10.1016/j.jad.2007.09.003

[14] J. Mirsky, R. Kohn, I. Levav, A. Grinshpoon and A. M. Ponizovsky, "Psychological Distress and Common Mental Disorders among Immigrants: Results from the Israeli-Based Component of the World Mental Health Survey,” Journal of Clinical Psychiatry, Vol. 69, No. 11, 2008, pp. 1715-1720. doi:10.4088/JCP.v69n1106 